\title{
The beginning of a new era: East meets West more comfortably regarding lymphadenectomy for gastric cancer - Japan will finally drop the surgery-alone arm in its pursuit of a multimodal treatment strategy
}

\author{
YASUHIRO KODERA \\ Department of Surgery II, Nagoya University Graduate School of Medicine, 65 Tsurumai-cho, Showa-ku, Nagoya, Aichi 466-8550, Japan
}

At the 79th Japan Gastric Cancer Association Meeting hosted by Dr. Yoshitaka Yamamura of Aichi Cancer Center, two long-awaited Phase III data sets from Japanese multiinstitutional study groups were unveiled for the first time in their home country. Accordingly, two symposia had been planned to feature each of these important results, and they were delivered successively on the morning of March 2, 2007.

One of the symposia, entitled "Lymphadenectomy for advanced gastric cancer with potential for cure," was planned by the author with the intention of informing the domestic audience of the state of the art regarding the much debated issue of the extent of lymphadenectomy for gastric cancer. This symposium was chaired by Dr. Takashi Aiko of Kagoshima University and Dr. Mitsuru Sasako of the National Cancer Center, Tokyo, two of the most influential figures in the development of gastric cancer surgery in Japan.

Although an international consensus regarding the prognostic impact of D2 lymphadenectomy (dissection of lymph node stations along the hepatic and splenic arteries and around the celiac axis in addition to the perigastric lymph nodes, not to mention a few other details) had been lacking [1], this procedure remained a standard in Japan based on reasonably high numbers of survivors among the patients who had metastases to these relatively distant lymph node stations. Following what seemed to be a success, the Japanese surgeons went on to extend the range of lymphatic clearance because they thought this was the only rational surgical solution to improve cure rates for the advanced disease. Paraaortic lymph nodes had been considered the final station before lymphatic spread develops into a systemic and incurable disease, and sporadic pilot studies to test the feasibility of extensively dissecting these

Offprint requests to: Y. Kodera nodes in addition to D2 had been performed throughout the country since the 1980s. Surgeons actively participating in these attempts often deliberately described this procedure as easily performed and effective with no increase in mortality; and domestic surgical meetings at the time were filled with arrays of videos in which the aorta and inferior vena cava were mercilessly exposed. In 1995, Sasako et al. made a rational approach to prove the prognostic value of this procedure and launched the Japan Clinical Oncology Group (JCOG) 9501 study, a Phase III trial comparing D2 versus D2 plus paraaortic lymph node dissection, with overall survival the primary endpoint. The final results of this trial were now available and were presented at this symposium.

During a short session of 90 minutes, only papers describing or analyzing results of Phase III trials were selected for presentation. Given that JCOG9501 was the first study of its kind in Japan, the presenters in the symposium inevitably included several foreign participants, but the presentations were translated into Japanese, the official language of the congress, to ensure full understanding by the domestic audience. The core presentations were preceded by an informative, beautifully presented review by Dr. Shoji Natsugoe of Kagoshima University describing the history of clinical trials on lymphadenectomy for gastric cancer carried out in various countries and aptly pointing to where we all stood prior to this meeting.

The paper by Dr. Scott Hundahl presented the earlier generation of clinical trials, all of which failed to prove the superiority of D2 dissection over D1. Dr. Hundahl analyzed the Dutch D1 versus D2 trial [2] from the viewpoint of "at risk" node stations left in situ and, to the expectation of the Japanese audience, reached a conclusion that high-quality surgery could affect survival even among the population registered for this negative study. Dr. Hundahl established a Maruyama index, which is the sum of the incidence of metastasis as a percentage (as given in a database compiled at the 
National Cancer Center Hospital, known as the Maruyama computer program) among the first- and second-tier lymph node stations, which were not dissected during that particular surgery [3]. This index circumvents the issues of noncompliance and contamination, which were factors confounding the lymphadenectomy clinical trials [4], and enables direct comparison albeit nonrandomized between good-quality surgery versus bad quality surgery. Among the population entered in the Dutch trial, the survival of patients with a Maruyama index of $<5$ (a group undergoing high-quality surgery) was found to be significantly better [5]. We have seen that accurately performing D2 dissection calls for detailed knowledge of the anatomy of regional lymph nodes. Dr. Hundahl recommended that Western surgeons forget about the notion of $\mathrm{D}$ numbers, which for them is inevitably based on blurred memories of the anatomy and, instead, refer to the Maruyama computer program prior to or even during surgery so as to have explicit knowledge of which lymph nodes to dissect during that particular surgery to fulfill the criteria of "good surgery." A synopsis of this presentation is published in this issue of Gastric Cancer. Of particular importance is the failure analysis made possible through the extensive autopsy data, indicating that surgery with a Maruyama index $<5$ led to a remarkably reduced incidence of local recurrence [6].

Dr. Chew-Wun Wu of the Taiwan Veterans Hospital presented data from their single-institutional D1 versus D2 trial recently published in Lancet Oncology [7]. Dr. Sasako, the chairperson, made clear that what the presenter referred to as D3 in his publication as well as the presentation at this meeting is equivalent to D2 by the current edition of the Japanese Classification of Gastric Carcinoma. The Japanese audience appreciated hearing details of what turned out to be the only trial so far that showed survival benefit of D2 dissection, their standard of care. Dr. Wu revealed that the proportion of patients who were up-staged by more detailed pathological examination as a result of extended lymphadenectomy was minimal [8] and attempted to convince the audience that the prognostic benefit of D2 dissection in their trial is not merely a consequence of stage migration.

Finally, Dr. Takeshi Sano of the National Cancer Center, Tokyo, presented the final survival analysis of the JCOG9501 study. This study had already revealed obvious shortcomings of the paraaortic dissection in terms of a longer operating time and greater blood loss, leading to twice the incidence of blood transfusion, with a moderate increase in postoperative complications [9]. Survival analysis showed no difference between the groups, and the superextended lymphadenectomy, once a cutting-edge technique considered to strengthen the Japanese concept of radical surgery, was shown not to be rewarding when applied prophylactically to a population with $>$ T2b, N0-2, M0 lesions. Findings from the subset analyses were more intriguing. There was a clear survival advantage of paraaortic lymph node dissection among those who were found after histological evaluation to have been $\mathrm{pN} 0$, whereas the lymphadenectomy had a significant adverse effect for patients with histologically node-positive disease for whom the procedure had originally been intended.

These presentations were followed by comments from two selected discussants. Dr. Toshimasa Tsujinaka of the Osaka Medical Center compared the three trials and pointed out a striking resemblance in the incidence of locoregional recurrence at around $20 \%$ among patients accrued for the JCOG trial and those allocated to the D2 arm of the Taipei trial. This pattern of failure was observed in $29 \%$ of Dutch D2 patients, 30\% of Taiwanese D1 patients, and $41 \%$ of Dutch D1 patients. These data suggest that D1 surgery is not sufficient for local control even in the hands of experienced surgeons, and the quality of the Dutch D2 surgery had been insufficient despite their effort, as documented also by the report on noncompliance and calculation of the Maruyama index. Dr. Tsujinaka also calculated the survival benefit that could have been anticipated in each trial by multiplying the rate of metastases to the lymph nodes to be dissected only in the experimental arm (second-tier lymph nodes in the Dutch and Taipei trials and the paraaortic lymph nodes for the JCOG9501 trial) and the survival rate of patients who had metastasis to that group of lymph nodes. Taipei trial did fulfill this expectation, whereas the Dutch trial failed, presumably because of the high mortality and rate of noncompliance. Survival benefit anticipated for the JCOG trial, on the other hand, had been very small, suggesting that the trial had been, in a way, destined to be a negative study.

Although Dr. Tsujinaka and several Japanese participants considered the Maruyama index ingenious, Dr. Han-Kwang Yang of Seoul National University pointed out, rationally, that the database used for the Maruyama computer program, consisting of data from 3843 patients operated on at the National Cancer Center in Tokyo [10], should now be updated and expanded to include data from other leading institutions because the number of patients in some subgroups stratified by numerous clinical variables tended to be small. Dr. Yang pointed out the weakness of the $\mathrm{D} 2$ dissection in that only one trial has ever proved a survival benefit with this procedure [7]. However, it is not practical, in his opinion, to plan yet another D1 versus D2 trial in a country where D2 dissection is safely performed with satisfactory outcome, whereas the same study performed by a group not familiar with D2 is likely to follow the footsteps of the Dutch and Medical Research 
Council (MRC) trials [11]. This is a view that was shared by most Japanese surgeons in the past who, instead, planned and conducted the JCOG9501 trial.

Perhaps we have a combination of Phase III evidence today that may satisfy everyone for the time being. General surgeons in the West may remain content with D1 dissection because it is safer; and, after all, there is only one set of evidence supporting D2 dissection [7]. Surgery in this case obviously must be regarded as a component of a multimodal treatment strategy. On the other hand, advocates of D2 dissection can feel more comfortable because, at last, there is one set of Phase III evidence to justify their surgery, and there is one other piece of evidence that a greater effort is not necessary. Those who are reluctant to discard paraaortic lymph node dissection should proceed to test the impact of this major surgery in a population suffering from paraaortic lymphadenopathy or bulky node metastases, perhaps after neoadjuvant chemotherapy in a clinical trial setting. State-of-the-art treatment for advanced gastric cancer is, as Dr. Tsujinaka wisely concluded, high-quality surgery and adequate adjuvant therapy.

The second symposium of the morning, entitled "Postoperative adjuvant chemotherapy for gastric carcinoma: beginning of a new era," was chaired by Dr. Tsuneo Sasaki of Tokyo Metropolitan Komagome Hospital and the author. The symposium was centered around the ACTS-GC study, a Phase III study comparing eight courses of oral agent S-1 [12] $\left(80 \mathrm{mg} / \mathrm{m}^{2}\right.$ per a day for 4 weeks followed by 2 weeks of rest) given postoperatively versus treatment by surgery alone for stage II-IIIB gastric cancer.

Since the late 1980s, several JCOG Phase III trials had been performed to prove the benefit of postoperative adjuvant chemotherapy following D2 dissection over surgery alone [13-15], but all attempts failed owing possibly to suboptimal selection criteria, inadequate study designs leading to small sample size, or unavailability of active cytotoxic agents. In the meantime, some important Phase III evidence was reported in the West. Both the Intergroup 0106 study [16] and the MAGIC trial [17] have been conceived in Japan as a reflection of the fact that postoperative adjuvant chemoradiation or intense perioperative chemotherapy partially compensated for poor local control as a consequence of suboptimal surgery. With an opinion that this evidence cannot be shared unconditionally in a country where sufficient local control has been achieved by radical surgery, the Japanese Gastric Cancer Treatment Guidelines continue to recommend treatment with D2 dissection alone for stage I-III disease and advise that adjuvant therapy be used only in the clinical trial setting. Nevertheless, postoperative adjuvant chemotherapy had been utilized as a general practice by most surgeons in Japan, reflecting an old tradition based on clinical trials performed during the 1960s that would now be considered faulty and outdated [18].

ACTS-GC, presented by Dr. Taira Kinoshita of National Cancer Center East, Kashiwa, was a clear breakthrough for this frustrating situation; and treatment by the single agent $\mathrm{S}-1$ provided a risk reduction by $32 \%$ in this pivotal trial that accrued 1059 patients. The difference in overall survival was highly significant at the first interim analysis performed 1 year after termination of the accrual. The $P$ value for the difference in overall survival of all 1034 eligible patients was $P=$ 0.0008 , which was lower than $P=0.0011$, the criterion for stopping the trial (O'Brien Fleming stopping boundary). Adverse reactions were mild and manageable, and there were no treatment-related deaths. Professor Kinoshita confidently concluded that postoperative S-1 will be a new standard of care for stage II-IIIB gastric carcinoma in Japan.

Dr. Toshifusa Nakajima of the Cancer Institute Hospital, Tokyo, who had painstakingly organized several of the past JCOG trials, has finally had a last laugh in the N-SAS-GC study, which he himself presented prior to the talk by Dr. Kinoshita. In his trial, patients with pathologically $\mathrm{T} 2$ and node-positive ( $\mathrm{pN} 1$ or $\mathrm{pN} 2)$ cancer were randomized to receive postoperative oral UFT or treatment with surgery alone. Dr. Nakajima selected this particular population based on subset analyses of past Phase III studies, and the UFT treatment was intensified by dose escalation to $360 \mathrm{mg} / \mathrm{m}^{2}$ to be given 5 days a week with 2 days of rest instead of the usual dose of $300-400 \mathrm{mg} /$ body given every day. Adverse reactions were mild and manageable, and the difference in survival at the second interim analysis met the stopping boundary of $P=0.0192$. This is the first positive result in support of postoperative adjuvant chemotherapy over surgery alone in Japan and could have been considered more seriously had ACTS-GC not been planned as a successor to this trial. In reality, only 190 patients were registered in this study (188 eligible), whereas accrual of 488 had been planned in the original design. Given this small size and the limits in the eligibility criteria, N-SAS-GC had not been considered sufficient on its own to revise the aforementioned recommendation in the Gastric Cancer Treatment Guidelines.

After these presentations, two discussants gave their opinions regarding the two trials and perspectives for future clinical trials in Japan. Dr. Hiroya Takiuchi, a medical oncologist from Osaka Medical College, commended ACTS-GC as a milestone in the history of adjuvant treatment for gastric cancer in Japan. He then looked at subgroups in greater detail and pointed out several issues that could be addressed in future trials. For stage II disease, D2 dissection followed by S-1 for 
1 year resulted in an excellent 3-year overall survival of $90.7 \%$, and there is little room for further improvement. He proposed a trial to show the noninferiority of a regimen in which postoperative S-1 is continued for 6 months instead of 12 months, as seen with the adjuvant treatment of other cancer types. He doubted the value of a regimen tested in the N-SAS-GC study (UFT $360 \mathrm{mg} / \mathrm{m}^{2}$ on days $1-5$ every week) because there was no difference in the incidence of severe anorexia and diarrhea, the toxicities that most often lead to cessation of the treatment, between UFT at this dosage and S-1. He preferred to use UFT in the original $250 \mathrm{mg} / \mathrm{m}^{2}$ dosage, whose marginal prognostic impact was suggested in lung cancer. UFT at this dosage could then be selected for patients who found S-1 to be too toxic. On the other hand, survival of stage IIIA and IIIB subgroups could be improved by using S-1 in combination with other drugs or by adding neoadjuvant chemotherapy. Several study groups are now planning to test the feasibility of combination regimens, such as S-1/cisplatin [19] and S-1/docetaxel [20] in the postoperative adjuvant setting. The prognostic impact of S- $1 /$ cisplatin in the neoadjuvant setting is currently being tested in another JCOG study. Dr. Takiuchi's talk was then extended to cover the treatment strategy for patients who failed during or after adjuvant treatment with S-1. His future perspectives included the introduction of molecular targeting agents to adjuvant therapy, and he concluded that surgeons and medical oncologists should collaborate to make further breakthroughs in this field of research.

Another discussant, Dr. Junichi Sakamoto of Nagoya University, criticized the way ACTS-GC was analyzed and publicized. Interim analyses had been planned at 1 and 3 years after the termination of patient accrual. At the first interim analysis, the $P$ value for the difference in overall survival of all 1034 eligible patients $(P=0.0008)$ was lower than the O'Brien Fleming stopping boundary $(P=0.0011)$, but for all patients randomized it was $P=0.0016$ and exceeded the boundary. Dr. Sakamoto argued that the early stopping rule should not have been applied at this time, given that data analysis in such a critical situation should have been based on all patients randomized. Moreover, the early stoppage was announced unofficially in June 2006 (6 months after the planned interim analysis) only among institutions that took part in the trial. Dr. Sakamoto pointed out that the time and method of publication should have been more discreetly decided. He also criticized the decision by the organizers to terminate the planned biannual follow-up of the patients. He mentioned a Cancer and Leukemia Group B 9633 study as an example that the magnitude of risk reduction diminished substantially after a longer follow-up, as a result of which a carboplatin/paclitaxel regimen of postoperative chemotherapy for non-small-cell lung cancer was deleted from the NCCN Practice Guidelines in Oncology. Dr. Sakamoto postulated the need for a longer follow-up before the results of ACTS-GC could be considered definitely positive and concluded that time is not ripe to revise the Gastric Cancer Treatment Guidelines.

Dr. Mitsuru Sasako, the principal investigator of ACTS-GC, confronted the argument with the following comments. In short, he and other investigators had decided after a vigorous discussion to accept the advice of the Data and Safety Monitoring Committee, which included a biostatistician, to stop the trial and publicize the results. He considered the Committee decision to have been adequate because with the predictive power of $99.3 \%$ the possibility that the difference in survival loses significance through longer follow-up can practically be dismissed as negligible. He then explained that the interim analysis was performed as originally planned. The problem was that, admittedly, there was a large time lag before the analysis could be started. The decision to terminate the biannual follow-up reflected the intention of the sponsor, who would not continue paying costs for the follow-up once a conclusion had been reached. On the other hand, when the early stopping rule was applied to a Phase III study, top-class journals tend to request a longer follow-up and are not really interested in survival data obtained at the time of the interim analysis. The Data and Safety Monitoring Committee thus recommended another 6 months of follow-up, and analysis at that time point became the final survival data presented during the current symposium. Dr. Sasako concluded that amending the protocol regarding a schedule for the final survival analysis, as observed in this trial, is by no means uncommon when a randomized controlled trial is stopped as a consequence of the interim analysis and should not be considered a protocol violation.

Because these discussions consumed an unexpected amount of time, the remaining presentations of this symposium on various themes that may be of help in designing future trials for adjuvant therapies were treated as lectures, and there was no allocation of time for questions or discussion. Dr. Akira Tsuburaya of Kanagawa Cancer Center reported on his pivotal study whose aim is to test a novel adjuvant chemotherapy regimen based on a compelling theory by Norton and Simon [21]; it consists of weekly administration of paclitaxel for 3 months followed by oral S-1 for 3 months [22]. Several scientific backgrounds to this unique sequential regimen were explained in detail. Three other treatment arms included chemotherapy with S-1 alone, UFT alone, and paclitaxel followed sequentially by UFT to constitute a $2 \times 2$ factorial design [23]. This design 
enables a comparison between oral fluoropyrimidine preceded and not preceded by paclitaxel and another comparison between UFT and S-1. The planned sample size is 1500 , of which 911 patients have been accrued as of March 2007.

Dr. Mitsuru Sasako of the National Cancer Center had a role in discussing the potential of irradiation as a component of future adjuvant therapy for advanced gastric cancer. He repeated the Japanese interpretation of the Intergroup 0106 study [16] that postoperative adjuvant chemoradiation merely compensated for the poor local control as a consequence of suboptimal surgery and may not be as effective after a D2 dissection. Subset analyses of the 0106 study stratified according to the extent of lymphadenectomy revealed that the difference between the treatment arm and active control was actually nullified in the D2 dissection group. Late renal toxicity, which is reported to emerge a few years after the radiotherapy, was of concern. He then mentioned a Korean observational study in which benefit of postoperative chemoradiotherapy was seen even among those treated by D2 dissection [24], but this report was criticized as being typically biased owing to nonrandomization. He concluded that although the effect of chemoradiotherapy after D2 dissection could not be denied its priority for further exploration is low compared with other strategies that wait in line as candidates for the next Phase III trials in Japan.

Finally, Dr. Masanori Terashima of Fukushima Medical University updated the audience with a detailed and informative survey of various techniques to tailor patients for appropriate therapies. The subject of his lecture ranged from pharmacogenetic and pharmacogenomic to proteomic approaches for predicting efficacy and toxicity by various adjuvant treatments. In particular, he made a brief survey of investigations of three major enzymes related to the metabolism of 5-FU. He then revealed a painstaking process that is required for clinical validation of initial findings from modern technology, such as the DNA microarray approach.

To close this report, the author believes that the debate regarding the extent of lymphadenectomy for gastric cancer is almost over. There are two standards: D1 for general surgeons in low-volume hospitals and D2 for surgeons in Far Eastern countries and expert surgeons at specialized centers. Adequate adjuvant therapies should accompany surgery, but the regimen used for the treatment should vary according to the stage of the cancer and the quality of the surgery applied. Now that the long and painful "dark age" of comparing an adjuvant chemotherapy with observation alone is over in Japan, arrays of trials to test new drugs and strategies will follow and this, indeed, will be the "beginning of a new era." The next edition of Gastric Cancer Treatment Guidelines complied by the Japan Gastric Cancer
Association might well be rewritten to reflect or impinge directly on some of the findings presented at these symposia. In the meantime, final publications that officially describe the results of JCOG9501, N-SAS-GC, and ACTS-GC studies are eagerly awaited.

\section{References}

1. Kodera Y, Schwarz RE, Nakao A. Extended lymph node dissection in gastric carcinoma: where do we stand after the Dutch and British randomized trials? J Am Coll Surg 2002;195:85564.

2. Bonenkamp JJ, Hermans J, Sasako M, van de Velde CJ, Welvaart $\mathrm{K}$, Songun I, et al. Extended lymph-node dissection for gastric cancer. N Engl J Med 1999;340:908-14.

3. Hundahl SA, Macdonald JS, Benedetti J, Fitzsimmons T, Southwest Oncology Group, Gastric Intergroup. Surgical treatment variation in a prospective, randomized trial of chemotherapy in gastric cancer: the effect of undertreatment. Ann Surg Oncol 2002;9:278-86.

4. Bonenkamp JJ, Hermans J, Sasako M, van de Velde CJH. Quality control of lymph node dissection in the Dutch randomized D1 and D2 lymph node dissection for gastric cancer. Gastric Cancer 1998;1:152-9.

5. Peeters KC, Hundahl SA, Kranenbarg EK, Hartgrink H, van de Velde CJ. Low Maruyama index surgery for gastric cancer: blinded reanalysis of the Dutch D1-D2 trial. World J Surg 2005; 29:1576-84.

6. Hundahl SA, Peeters KCMJ, Kranenbarg EK, Hartgrink H, van de Velde CJH. Improved regional control and survival with "low Maruyama Index" surgery in gastric cancer: autopsy findings from the Dutch D1-D2 Trial. Gastric Cancer 2007;10:84-6.

7. Wu CW, Hsiung CA, Lo SS, Hsieh MC, Chen JH, Li AF, et al. Nodal dissection for patients with gastric cancer: a randomized controlled trial. Lancet Oncol 2006;7:309-15.

8. Wu CW, Hsiung CA, Lo SS, Hsieh MC, Chen JH, Li AF, et al. Stage migration influences on stage-specific survival comparison between D1 and D3 gastric cancer surgeries. Eur J Surg Oncol 2005;31:153-7.

9. Sano T, Sasako M, Yamamoto S, Naashimoto A, Kurita A, Hiratuska M, et al. Gastric cancer surgery: morbidity and mortality results from a prospective randomized controlled trial comparing D2 and extended para-aortic lymphadenectomy — Japan Clinical Oncology Group study 9501. J Clin Oncol 2004;22:2759-61.

10. Droste K, Bollschweiler E, Waschulzik T, Schutz T, Engelbrecht $\mathrm{R}$, Maruyama K, et al. Prediction of lymph node metastasis in gastric cancer patients with neural networks. Cancer Lett 1996; 109:141-8.

11. Cuschieri A, Weedens S, Fielding J, Bancewicz J, Craven J, Joypaul V, et al. Patient survival after D1 and D2 resections for gastric cancer: long-term results of the MRC randomized surgical trial. Br J Cancer 1999;79:1522-30.

12. Sakata Y, Ohtsu A, Horikoshi N, Sugimachi K, Mitachi Y, Taguchi T. Late phase II study of novel oral fluoropyrimidine anticancer drug S-1 (1 M tegafur- $0.4 \mathrm{M}$ gimestat- $1 \mathrm{M}$ otastat potassium) in advanced gastric cancer patients. Eur J Cancer 1998;34: 1715-20.

13. Nakajima $T$, Nashimoto A, Kitamura M, Kito $T$, Iwanaga $T$, Okabayashi K, et al. Adjuvant mitomycin and fluorouracil followed by oral uracil plus tegafur in serosa-negative gastric cancer: a randomised trial - Gastric Cancer Surgical Study Group. Lancet 1999;354:273-7.

14. Nashimoto A, Nakajima T, Furukawa H, Kitamura M, Kinoshita T, Yamamura Y, et al. Randomized trial of adjuvant chemotherapy with mitomycin: fluorouracil and cytosine arabinoside followed by oral fluorouracil in serosa-negative gastric cancer: 
Japan Clinical Oncology Group 9206-1. J Clin Oncol 2003;21: 2282-7.

15. Miyashiro I, Furukawa H, Sasako M, Yamamoto S, Nashimoto A, Nakajima T, et al. No survival benefit with adjuvant chemotherapy for serosa-positive gastric cancer: randomized trial of adjuvant chemotherapy with cisplatin followed by oral fluorouracil (UFT) in serosa-positive gastric cancer - Japan Clinical Oncology Group 9206-2. In: Proceedings of the Gastointestinal Cancers Symposium, 2004.

16. Macdonald JS, Smalley SE, Benedetti J, Hundahl SA, Estes NC, Stemmermann GN, et al. Chemoradiotherapy after surgery compared with surgery alone for adenocarcinoma of the stomach or gastroesophageal junction. N Engl J Med 2001;345:723-30.

17. Cunningham D, Allum WH, Stenning SP, Thompson JN, van de Velde CJ, Nicolson M, et al. Perioperative chemotherapy versus surgery alone for resectable gastroesophageal cancer. N Engl J Med 2006;355:11-20.

18. Imanaga $\mathrm{H}$, Nakazato $H$. Result of surgery for gastric cancer and effect of adjuvant mitomycin $\mathrm{C}$ on cancer recurrence. World $\mathrm{J}$ Surg 1977;2:213-21.

19. Koizumi W, Tanabe S, Saigenji K, Ohtsu A, Boku N, Nagashima F, et al. Phase I/II study of S-1 combined with cisplatin in patients with advanced gastric cancer. Br J Cancer 2003;89: 2207-12.
20. Yoshida K, Nimomiya M, Takakura N, Hirabayashi N, Takiyama W, Sato Y, et al. Phase II study of docetaxel and S-1 combination therapy for advanced or recurrent gastric cancer. Clin Cancer Res 2006;12:3402-7.

21. Simon R, Norton L. The Norton-Simon hypothesis: designing more effective and less toxic chemotherapeutic regimens. Nat Clin Pract Oncol 2006;3:406-7.

22. Kobayashi M, Tsuburaya A, Nagata N, Miyashita Y, Oba K, Sakamoto J. A feasibility study of sequential paclitaxel and S-1 (PTX/S-1) chemotherapy as postoperative adjuvant chemotherapy for advanced gastric cancer. Gastric Cancer 2006;9:114-9.

23. Tsuburaya A, Sakamoto J, Morita S, Kodera Y, Kobayashi M, Miyashita Y, et al. A randomized phase III trial of postoperative adjuvant oral fluoropyrimidine versus sequential paclitaxel/oral fluoropyrimidine; and UFT versus S-1 for T3/T4 gastric carcinoma: the Stomach Cancer Adjuvant Multi-institutional Trial Group (SAMIT) trial. Jpn J Clin Oncol 2005;35: $672-5$.

24. Kim S, Lim DH, Lee J, Kang WK, MacDonald JS, Park CH, et al. An observational study suggesting clinical benefit for adjuvant postoperative chemoradiation in a population of over 500 cases after gastric resection with D2 nodal dissection for adenocarcinoma of the stomach. Int J Radiat Oncol Biol Phys 2005; 63:1279-85. 